\title{
Clinical characteristics vary between women and men in 67,524 patients with moderate obesity preoperative for laparoscopic adjustable gastric Band (LAGB)
}

\begin{abstract}
Background: As of 2018, LAGB represents $<2 \%$ of weight loss operations, but with excellent long-term outcomes in selected patients. Thus, every clinical insight can help in selecting LAGB patients and planning surgery. While more women than men undergo bariatric surgery, differences between the sexes in obesity-related clinical conditions preoperatively among patients seeking LAGB have not been investigated. The objective of this study was to identify variations in obesity-related co-morbidities among women and men who chose laparoscopic adjustable gastric band (LAGB).
\end{abstract}

Methods: Pre-operative data on 53,292 women and 14,222 men undergoing LAGB were examined retrospectively from the Surgical Review Corporation's Bariatric Outcomes Longitudinal Database (BOLD)

Results: Women were more frequently African American and on Medicaid while men were more frequently Caucasian insured with Medicare. Men were older, heavier, and used alcohol, tobacco, and illicit substances more frequently. Men had higher rates of cardiovascular and pulmonary disease, diabetes, gout, impaired functional status, abdominal hernia, and liver disease. Women had higher rates of asthma, cholelithiasis, urinary incontinence, panniculitis, and mental health.

Conclusion: Pre-operative characteristics of LAGB patients vary by sex. This clinical knowledge may aid physicians and surgeons in facilitating anticipatory management of patients with moderate obesity.

Keywords: obesity, adjustable gastric band, bariatric surgery, metabolic surgery, obesity co-morbidities, laparoscopy
Volume 10 Issue 4 - 2020

\section{Christopher Bashian, Jandie Schwartz, Luke Perry, Gus J Slotman}

Department of Surgery, Inspira Health Network, USA

Correspondence: Gus J Slotman, Director of Clinical Research, Inspira Health Network, Clinical Professor of Surgery RowanSOM, I 505 W Sherman Avenue Suite B,Vineland, NJ 08360, USA, Tel 856-64I-8635, Fax 856-64I-8636, Email Slotmng@ihn.org

Received: July 21, 2020 | Published: August 21, 2020
Abbreviations: PVD, peripheral vascular disease; HTN, hypertension; MHD, mental health diagnoses; IFS, impaired functional status

\section{Introduction}

As of 2018, LAGB represents $<2 \%$ of weight loss operations, but continues to effect excellent long-term outcomes in selected patients. ${ }^{1}$ For patients with moderate obesity, every clinical insight can help in selecting LAGB patients and planning surgery. While more women than men undergo bariatric surgery, differences between the sexes in obesity-related clinical conditions pre-operatively among patients seeking LAGB have not been investigated. We have reported previously that the pre-operative clinical characteristics of laparoscopic Roux-en-Y gastric bypass (LRYGB) patients, and of patients undergoing biliopancreatic diversion/duodenal switch (BPD/DS) differed significantly women versus men. ${ }^{2-4}$ However, whether or not such variations by sex are clinically important also in the moderately obese population of patients who choose LAGB is unknown. The objective of this study was to identify variations in demographics, age, BMI, and the incidence of obesity co-morbidities between women versus men with moderate obesity who were preoperative for LAGB.

\section{Methods}

Pre-operative clinical data from 67,524 patients in the Surgical Review Corporation's Bariatric Outcomes Longitudinal Database (BOLD)5 who were about to undergo LAGB were examined retrospectively in two groups: women $(53,292)$ and men $(14,222)$. Weight, BMI, age, race, health insurance status, and the percent frequency of obesity co-morbidities were obtained from BOLD, including hypertension (HTN), angina, congestive heart failure (CHF), peripheral vascular disease (PVD), obstructive sleep apnea (OSA), obesity hypoventilation syndrome (OHS), asthma, ischemic heart disease (IHD), deep vein thrombosis/pulmonary embolism (DVT/PE), abdominal hernia, abdominal panniculitis, cholelithiasis, gastroesophageal reflux disease (GERD), liver disease, stress urinary incontinence (SUI), diabetes mellitus, gout, dyslipidemia, pseudotumor cerebri, back pain, lower extremity edema (LEE), musculoskeletal pain, mental health diagnoses (MHD), impaired functional status (IFS), depression, psychological impairment, alcohol use, substance abuse, tobacco use, and fibromyalgia. ${ }^{5}$

Statistical analysis: Continuous variables were analyzed using analysis of variance (ANOVA). Pair-wise comparisons were performed on the least squares means of the treatments calculated 
from the ANOVA model to find differences in the treatment groups. Distribution of obesity co-morbidities was examined by a general linear model with treatment in the model and modified for binomial distribution to account for dichotomous variables. ${ }^{6}$

\section{Results}

Demographics in moderately obese women and men are listed in Table 1. Female/male age $(45.2 \pm 12 / 48.2 \pm 12$ years $)$, weight $(119 \pm 19 / 147 \pm 25$ kilograms) (BMI $(44.6 \pm 7 / 46.2 \pm 7)$ health insurance (Medicaid 3.1\%/1.6\%, Medicare 7.1\%/9.9\%) $(\mathrm{p}<0.0001)$ and race (African-American $12.4 \% / 6.8 \%$, Caucasian $73.5 \% / 78.3 \%$, Hispanic $5.8 \% / 6.4 \%$, Asian $0.2 \% / 0.3 \%$, Other $7.8 \% / 8.1 \%$; $<<0.001$ ) varied significantly. Cardiopulmonary co-morbidities in moderately obese women and men are displayed in Table 2. Cardiovascular comorbidities angina, CHF, HTN, IHD, PVC $(\mathrm{p}<0.0001)$, and DVT/PE $(p<0.01)$ were more prevalent in males. Only lower extremity edema was increased in women. $(\mathrm{p}<0.01)$. OSA and OHS were higher in males, while asthma was more frequent in females $(\mathrm{p}<0.0001)$. Psychological and Behavioral co-morbidities in moderately obese women and men are tabulated in Table 3. Male alcohol use, tobacco and substance abuse, and unemployment were greater than female $(\mathrm{p}<0.0001)$ Women suffered depression, psychological impairment, and mental health diagnoses more often than men $(p<0.0001)$. Abdominal and hepatobiliary co-morbidities in moderately obese women and men are detailed in Table 4. Abdominal hernia and liver disease were higher among men $(p<0.0001)$. Females more frequently had GERD, abdominal panniculitis, cholelithiasis, and SUI $(p<0.0001)$. Endocrine and metabolic co-morbidities in moderately obese women and men are demonstrated in Table 5. Diabetes, gout, and dyslipidemia were increased in men, while pseudotumor cerebri was higher in women $(\mathrm{p}<0.0001)$. Somatic co-morbidities in moderately obese women and men are presented in Table 6. Males more frequently had impairments in functional status, whereas females more often had fibromyalgia and back pain $(\mathrm{p}<0.0001)$ Musculoskeletal pain did not vary between males and females (p 0.3708). Overall, females carried 12 weightrelated co-morbidities more frequently than did males: abdominal panniculitis, cholelithiasis, GERD, stress urinary incontinence, asthma, back pain, fibromyalgia, pseudotumor cerebri, mental health diagnosis, depression, psychological impairment $(\mathrm{p}<0.0001)$ and lower extremity edema $(\mathrm{p}<0.01)$. Seventeen obesity co-morbidities were increased in men: abdominal hernia, liver disease, OHS, OSA, angina, HTN, CHF, IHD, PVD, dyslipidemia, diabetes, gout, impaired functional status, alcohol use, tobacco use, substance abuse, and unemployment $(\mathrm{p}<0.0001)$ and DVT/PE $(\mathrm{p}<0.01)$.

Table I Demographics in women and men with moderate obesity

\begin{tabular}{|c|c|c|c|c|c|c|c|c|c|c|}
\hline & Age & $\begin{array}{l}\text { Weight } \\
\text { (kg) }\end{array}$ & BMI & $\begin{array}{l}\text { African } \\
\text { American }\end{array}$ & Caucasian & Hispanic & Asian & Other & Medicaid & Medicare \\
\hline Women & $45.2 \pm 12$ & $119 \pm 19$ & $44.6 \pm 7$ & $12.40 \%$ & $73.50 \%$ & $5.80 \%$ & $0.20 \%$ & $7.80 \%$ & $3.10 \%$ & $7.10 \%$ \\
\hline Men & $48.2 \pm 12$ & $147 \pm 25$ & $46.2 \pm 7$ & $6.80 \%$ & $78.30 \%$ & $6.40 \%$ & $0.30 \%$ & $8.10 \%$ & $1.60 \%$ & $9.90 \%$ \\
\hline$p$-value & $<0.0001$ & $<0.0001$ & $<0.0001$ & $<0.001$ & $<0.001$ & $<0.001$ & $<0.001$ & $<0.001$ & $<0.0001$ & $<0.0001$ \\
\hline
\end{tabular}

Table 2 Cardiopulmonary co-morbidities in women and men with moderate obesity

\begin{tabular}{|c|c|c|c|c|c|c|c|c|c|c|}
\hline & Angina & CHF & HTN & $\begin{array}{l}\text { Ischemic } \\
\text { heart } \\
\text { disease }\end{array}$ & $\begin{array}{l}\text { Peripheral } \\
\text { vascular } \\
\text { disease }\end{array}$ & $\begin{array}{l}\text { Lower } \\
\text { extremity } \\
\text { edema }\end{array}$ & DVT/ PE & Asthma & $\begin{array}{l}\text { Obstructive } \\
\text { sleep apnea }\end{array}$ & $\begin{array}{l}\text { Obesity } \\
\text { hypoventilation } \\
\text { syndrome }\end{array}$ \\
\hline Women & $1.83 \%$ & I.15\% & $52.15 \%$ & $2.83 \%$ & $0.79 \%$ & $23.32 \%$ & $2.51 \%$ & $16.42 \%$ & $34.33 \%$ & $1.37 \%$ \\
\hline Men & $3.35 \%$ & $3.07 \%$ & $67.09 \%$ & $8.71 \%$ & $1.51 \%$ & $22.17 \%$ & $2.94 \%$ & $10.65 \%$ & $56.65 \%$ & $2.17 \%$ \\
\hline p-value & $<0.0001$ & $<0.0001$ & $<0.0001$ & $<0.0001$ & $<0.0001$ & $<0.01$ & $<0.01$ & $<0.0001$ & $<0.0001$ & $<0.0001$ \\
\hline
\end{tabular}

Table 3 Psychological/behavioral co-morbidities in women and men with moderate obesity

\begin{tabular}{llllllll}
\hline & Depression & $\begin{array}{l}\text { Psychological } \\
\text { impairment }\end{array}$ & $\begin{array}{l}\text { Alcohol } \\
\text { abuse }\end{array}$ & $\begin{array}{l}\text { Substance } \\
\text { abuse }\end{array}$ & $\begin{array}{l}\text { Tobacco } \\
\text { abuse }\end{array}$ & $\begin{array}{l}\text { Mental health } \\
\text { diagnosis }\end{array}$ \\
\hline Women & $34.29 \%$ & $14.83 \%$ & $29.74 \%$ & $0.31 \%$ & $5.99 \%$ & $9.69 \%$ & $16.55 \%$ \\
Men & $20.63 \%$ & $10.01 \%$ & $34.58 \%$ & $0.57 \%$ & $8.09 \%$ & $5.82 \%$ & $21.64 \%$ \\
P-value & $<0.0001$ & $<0.0001$ & $<0.0001$ & $<0.0001$ & $<0.0001$ & $<0.0001$ & $<0.0001$
\end{tabular}

Table 4 Abdominal/hepatobiliary co-morbidities in women and men with moderate obesity

\begin{tabular}{lllllll}
\hline & GERD & $\begin{array}{l}\text { Abdominal } \\
\text { hernia }\end{array}$ & $\begin{array}{l}\text { Abdominal } \\
\text { panniculitis }\end{array}$ & Cholelithiasis & $\begin{array}{l}\text { Stress urinary } \\
\text { incontinence }\end{array}$ & $\begin{array}{l}\text { Liver } \\
\text { disease }\end{array}$ \\
\hline Women & $43.97 \%$ & $3.95 \%$ & $4.84 \%$ & $19.44 \%$ & $25.14 \%$ & $3.91 \%$ \\
Men & $36.66 \%$ & $6.63 \%$ & $3.36 \%$ & $7.03 \%$ & $3.45 \%$ & $5.32 \%$ \\
P-value & $<0.0001$ & $<0.0001$ & $<0.0001$ & $<0.0001$ & $<0.0001$ & $<0.0001$ \\
\hline
\end{tabular}


Table 5 Endocrine/metabolic co-morbidities in women and men with moderate obesity

\begin{tabular}{lllll}
\hline & Diabetes & Gout & Dyslipidemia & $\begin{array}{l}\text { Pseudotumor } \\
\text { cerebri }\end{array}$ \\
\hline Women & $26.30 \%$ & $2.09 \%$ & $37.25 \%$ & $1.37 \%$ \\
Men & $37.82 \%$ & $6.75 \%$ & $48.24 \%$ & $0.64 \%$ \\
P-value & $<0.0001$ & $<0.0001$ & $<0.0001$ & $<0.0001$ \\
\hline
\end{tabular}

Table 6 Somatic co-morbidities in women and men with moderate obesity

\begin{tabular}{lllll}
\hline & $\begin{array}{l}\text { Back } \\
\text { pain }\end{array}$ & $\begin{array}{l}\text { Impaired } \\
\text { functional } \\
\text { status }\end{array}$ & Fibromyalgia & $\begin{array}{l}\text { Musculoskeletal } \\
\text { pain }\end{array}$ \\
\hline Women & $45.05 \%$ & $1.92 \%$ & $3.30 \%$ & $38.80 \%$ \\
Men & $42.74 \%$ & $2.89 \%$ & $0.55 \%$ & $21.70 \%$ \\
P-value & $<0.0001$ & $<0.0001$ & $<0.0001$ & 0.3708 \\
\hline
\end{tabular}

\section{Discussion}

The results of this study identify significant variation in the clinical characteristics of moderately obese women versus men who were pre-operative for LAGB. Women were more frequently African American and on Medicaid while men were more often Caucasian and on Medicare. Men were older, had higher BMI, used alcohol, drugs, and tobacco more frequently, and had higher rates of cardiovascular pathology (angina, CHF, hypertension, ischemic heart disease, PVD, DVT/PE), pulmonary dysfunction (obesity hypoventilation syndrome and obstructive sleep apnea), metabolic/endocrine conditions (diabetes, gout), abdominal hernia, liver disease, and impaired functional status. Among women, asthma, abdominal/hepatobiliary (cholelithiasis, stress urinary incontinence, abdominal panniculitis), and mental health issues/dysfunction were more frequent than in men. This advance clinical knowledge can complement clinical judgement of bariatric surgeons in selecting patients for LAGB and preparing them for surgery. In addition, since all surgeons of all specialties, not just bariatric surgeons, now must operate on morbidly obese patients, this advance clinical knowledge can increase clinical acumen in peri-operative patients with obesity and may facilitate anticipatory management. Understanding weight-related variation between women and men with moderate obesity thus may improve medical care and outcomes for these patients. Our review of the literature indicates that the statistically and clinically significant variations in baseline characteristics of moderately obese women versus men pre-surgical for LAGB have not been reported previously and are significant findings of this study. It is not surprising that males weighed more than females, but concurrent advanced male age may indicate that males choose bariatric surgery at a more progressive stage of obesity than females. Even though the gap in weight differences was small, these findings were still clinically significant. Advanced age in males with obesity is consistent with reports in the literature. ${ }^{7}$ Since men were also older and heavier than women in this study, that the maledominated categories appeared more frequently in patients covered by Medicare, including cardiovascular and pulmonary disease, diabetes, gout, impaired functional status, abdominal hernia, liver disease, tobacco abuse, alcohol abuse, and drug abuse, is consistent with the data. Conversely, with higher Medicaid coverage among LAGB women, female-dominated categories appeared more frequently in Medicaid insured, including asthma, cholelithiasis, SUI, panniculitis, and mental health. Thus, the prevalence of sex related obesity co-morbidities directly correlates to their prevalence in patients with Medicare versus Medicaid. Previously we reported variation between insurance carriers, with the highest obesity co-morbidities in Medicare and Medicaid, versus private insurance and Self-Pay. ${ }^{8,9}$ When organized by sex, more LAGB males were Caucasian, Hispanic, Asian, and Other. More females were African American. Caucasian men had the highest weight, BMI, and number of total co-morbidities. Previously we reported pre-operative variation by race in mixed sex populations undergoing open RYGB,${ }^{10}$ and by health insurance before LRYGB8, open RYGB9, and biliopancreatic diversion/duodenal switch. ${ }^{4}$ While Caucasian and African American women and men and Medicare and Medicaid subscribers in these investigations carried the highest BMI and obesity co-morbidities, when LAGB patients were analyzed by sex, Caucasian and Medicare men appeared to be at higher risk for excess weight and weight-related medical problems.

Men with moderate obesity in this study had significantly greater rates of all cardiovascular co-morbidities including HTN, angina, CHF, IHD, LEE, PVD and DVT/PE. These results are consistent with the literature documenting increased male cardiovascular risks over females in the general population. ${ }^{11-13}$ However, in the present investigation men with obesity not only had higher BMI than women, a health risk itself but also reported significantly more alcohol and tobacco abuse, both established risk factors for cardiopulmonary pathology. ${ }^{14}$ In addition to male sex, these factors may have contributed to the increased cardiovascular disease observed in men. Similar to the findings in our study, Weidner reported higher coronary artery disease in non-obese men than women, associated also with excessive alcohol consumption and smoking in these men. ${ }^{15}$ From this, one might speculate that in men with moderate obesity, behavioral misadventures contribute to their increased risk for coronary artery disease, angina, HTN, CHF, ischemic heart disease, and PVD.

Dyslipidemia is an independent risk factor for cardiovascular disease, and it is generally more frequent among men than women overall. ${ }^{16}$ This was confirmed in the present investigation of men and women with moderate obesity. Since male BMI in this study was higher than females, the results here support the concept of hyperlipidemia secondary to obesity as a direct risk factor for increased male risk for cardiopulmonary illnesses. Obese men had increased incidence of lower extremity edema in this study, which correlates with the literature stating that males overall have higher rates of venous insufficiency than women, and obesity further propagates its development. ${ }^{17}$ In this investigation, OSA and OHS affected men, at up to 1.65 times the female rate. These dramatic relative sex disparities contradict directly the findings of Beigh \& Jain, ${ }^{13}$ who reported metabolic syndrome in $29 \%$ of women and $23 \%$ of men with moderate obesity. The preoperative male/female variations in OSA and OHS rates in LAGB patients with mild to moderate obesity also are higher than those observed among patients undergoing LRYGB ${ }^{18}$ and $\mathrm{BPD} / \mathrm{DS} 4$, in spite of the higher absolute OSA and OHS percentages in the heavier latter populations. Approximately $25 \%$ adults with a BMI between 25 28 have at least mild obstructive sleep apnea, $30 \%$ of which are men and $15 \%$ women, supporting the differences between the sexes. ${ }^{19,20}$ The results here and the supporting literature identify increased OSA in men as a sex-related propensity that is exacerbated in incidence and severity by higher BMI and, possibly, by increased male smoking and drinking. Exacerbated relative male-to-female disparity in OSA and OHS among LAGB patients with moderate obesity is a new finding of this study. Our results also identified higher rates of diabetes in men versus women with moderate obesity. Increased male weight and BMI, which directly correlate to the development of Type 2 Diabetes, may be contributing factors. ${ }^{21}$ Variation in biology, culture, 
lifestyle, environment, and socioeconomic status impact males and females in development and clinical presentation of diabetes. Obese men nationwide have higher rates of diabetes than women, which is consistent with the findings of the present investigation..$^{22,23}$

The incidence of gout was increased significantly in LAGB men, compared with women. Gout is the most prevalent inflammatory arthritis in non-obese men and is associated with impaired quality of life. ${ }^{20} \mathrm{~A}$ direct relationship between increasing BMI and the incidence of gout has not been characterized in the general US population..$^{22,23}$ However, in the presence of obesity in the present investigation, both BMI and gout were increased significantly among LAGB men versus women. Pre-operative cholelithiasis was significantly greater in LAGB women than men studied here, consistent with historical trends and current data worldwide. Women are almost twice as likely to form gallstones as men, and most patients with gallstones in general are female and obese..$^{22}$ In a study of over 11,000 patients, by Sun et al. ${ }^{24}$ reported a positive association between increasing BMI and the incidence of cholelithiasis in both women and men. However, women developed cholelithiasis at a lower BMI compared with men. ${ }^{24}$ Sex and BMI are independent risk factors for cholelithiasis. Urinary incontinence affects more than 13 million women in the United States and profoundly impacts quality of life. Obesity is a strong risk factor for incontinence, consistent with the current results, one of multiple factors increasing the incidence among women. ${ }^{23,25}$ Women with incontinence in our study far outweighed the incidence in men, by nearly eight times. The weight-related problem of abdominal skin panniculitis was infrequent in this study population, but, nevertheless, affected a significant greater percentage of moderately obese women than it did men. Men had greater BMI, and abused tobacco, alcohol, and drugs more than women, all of which are well-known risk factors for infection. While fluctuations in female hormones which may contribute to obesity in women, ${ }^{26}$ the literature does not support hormonal changes as contributors to panniculitis. This is an important element to recognize as female sex may be an independent risk factor for panniculitis.

Women evaluated here experienced significantly greater GERD than did men, in contrast to the non-obese population in which GERD is approximately even between the sexes. ${ }^{27}$ Known risk factors for GERD are severe obesity, smoking, and alcohol abuse, ${ }^{28}$ all of which were increased in men in this study. From these results, one could expect a higher male GERD prevalence. Higher estrogen levels may act to inhibit the lower esophageal sphincter in women. ${ }^{17}$ The mechanism underlying increased GERD among women versus men with moderate obesity is not clear from the data. Psychological comorbidities in this study were diagnosed at higher rates among obese women for their male counterparts, consistent with prior publications. Mahoney and co-authors observed that women with obesity were more likely to report a history of anxiety and depression than men, and that they scored higher on psychiatric index testing preoperatively. ${ }^{29}$ Obese women in this study experienced greater somatic co-morbidities than men, with the exception of impaired functional status. This is interesting to note, as men would be expected to have higher somatic co-morbidities due to their higher BMI and weight. This has not been previously reported.

Men undergoing LAGB abused tobacco, alcohol, and drugs more than women. These lifestyle choices are significant as they may contribute to their overall obesity, and the development of multiple other co-morbidities. Hart et. al, hypothesized that obesity alone may lead to steatohepatitis, and the presence of fatty liver due to alcohol promotes obesity. ${ }^{15}$ This circular effect promotes dual detrimental effects on liver disease and obesity. This finding correlates with our data, in that increased alcohol abuse in men may be related to their higher weight and BMI. Increased alcohol abuse by LAGB men in this study also may have contributed to increased liver disease compared with women. Overall men consume alcohol more than women. ${ }^{30}$ The combined effects of alcohol and obesity may synergistically induce hepatocellular injury, inflammation, fibrosis and the development of cancer. ${ }^{31}$ Even at moderate doses alcohol exacerbates the pathological effects of obesity on the liver, as the threshold for hepatotoxic alcohol effects in obese populations is lower than for normal weight individuals..$^{28,32}$ These pathophysiologic interactions may be the mechanisms underlying increased male liver disease in this investigation. There were several limitations to this study. The data constraints of a retrospective investigation apply. Patients in this study represent a self-selected population who individually chose LAGB, and therefore our findings might not be applicable to all moderately obese surgical patients. Diagnosis of co-morbidities in BOLD was on a clinical diagnosis, rather than a pathological diagnosis. For example, liver biopsies were not required. Nevertheless, the significant clinical variation by sex reported here may be extrapolated to male and female patients with similar moderate obesity.

\section{Conclusion}

The pre-operative clinical characteristics of LAGB women and men varied significantly. Women were more frequently African American and on Medicaid while men were more often Caucasian and on Medicare. Before AGB, compared with women, men were older, had higher BMI, used alcohol, drugs, and tobacco more frequently, and had higher rates of cardiovascular pathology, obesity hypoventilation syndrome and obstructive sleep apnea, diabetes, gout, abdominal hernia, liver disease, and impaired functional status. Among women, asthma, cholelithiasis, stress urinary incontinence, abdominal panniculitis, and mental health issues/dysfunction were more frequent than for men. Although the LAGB patients in this study were self-selected, the significant variations by sex identified can be applied to other similar individuals with obesity. Since all surgeons now operate on patients with obesity, the advance clinical knowledge reported here can increase clinical acumen and may facilitate anticipatory management. Understanding variation in weight-related characteristics of men and women with moderate obesity may improve outcomes not only for surgery, but in medical management, as well.

\section{Acknowledgments}

None.

\section{Conflicts of interest}

The author declares that there is no conflict of interest.

\section{Funding}

This research did not receive grant funding from the public, commercial, or not-for-profit sectors.

\section{References}

1. ASMBS. Type 2 Diabetes and Obesity: Twin Epidemics. 2013.

2. Slotman G, Boulos P. In the Mega-Obese Weight Loss, Bmi and Resolution of Weight-Related Medical Problems after Biliopancreatic Bypass/Duodenal Switch Vary by Race: An Analysis of 1,673 Bold Database Patients. Surg Obes Relat Dis. 2015. 
3. Schwartz J, Bashian C, Kushnir L, et al. Variation in clinical characteristics of women versus men preoperative for laparoscopic Roux-en-Y gastric bypass: Analysis of 83,059 patients. In: American Surgeon. 2017.

4. Slotman G, Tamburrini DO. Battle of the super obese sexes: female versus male variation in pre-operative clinical characteristics among 1,673 surgical patients undergoing bilio-pancreatic bypass/duodenal switch (BPD/DS). Adv Obesity Weight Manag Control. 2019;9(2):47-51.

5. DeMaria EJ, Pate V, Warthen M, et al. Baseline data from American Society for Metabolic and Bariatric Surgery-designated Bariatric Surgery Centers of Excellence using the Bariatric Outcomes Longitudinal Database. Surgery for Obesity and Related Disorders. 2010;6(4):347-355.

6. SAS Institute Inc., Cary, NC U. Sas ${ }^{\circledR}$ 9.4. SAS Inst Inc, Cary, NC, USA; 2017.

7. U.S. Department of Health and Human Services. Managing Overweight and Obesity in Adults: Systematic Evidence Review from the Obesity Expert Panel. In: National Heart, Lung, and Blood Institute. 2013.

8. Blair K, Slotman G. Health Insurance Carrier Does Matter: Clinically Significant Variation in Weight-related Diagnoses for Medicaid versus Medicare versus Private Insurance versus Self Pay in 83,059 Morbidly Obese Patients. Adv Obes Weight Manag Control. 2018;8(3):178-183.

9. Davis M, Gomez J, Sheck C, et al. Variation in Weight and Obesity Comorbidities After Open Roux-en-Y Gastric Bypass by Health Insurance. JAMA Surg. 2017;152(1):105-109.

10. EA Racial variation in the distribution of demographics, body mass, and weight-related medical co-morbidities among the morbidly obese: Analysis of 5,389 bold database patients. Surg Endosc Other Interv Tech. 2014.

11. Naghavi M. Global, regional, and national burden of suicide mortality 1990 to 2016: systematic analysis for the Global Burden of Disease Study 2016. BMJ. 2019;364:194

12. Regitz-Zagrosek V, Oertelt-Prigione S, Prescott E, et al. Gender in cardiovascular diseases: Impact on clinical manifestations, management, and outcomes. Eur Heart J. 2016;37(1):24-34

13. Roeters Van Lennep JE, Westerveld HT, Erkelens DW, et al. Risk factors for coronary heart disease: Implications of gender. Cardiovasc Res. 2002;53(22):538-549.

14. Beigh SH, Jain S. Prevalence of metabolic syndrome and gender differences. Bioinformation. 2012;8(13):613-616.

15. Hart CL, Morrison DS, Batty GD, et al. Effect of body mass index and alcohol consumption on liver disease: Analysis of data from two prospective cohort studies. BMJ. 2010;340:c1240.

16. Miller M. Dyslipidemia and cardiovascular risk: The importance of early prevention. QJM. 2009;102(9):657-667.
17. Poirier P, Giles TD, Bray GA, et al. Obesity and cardiovascular disease: Pathophysiology, evaluation, and effect of weight loss: An update of the 1997 American Heart Association Scientific Statement on obesity and heart disease from the Obesity Committee of the Council on Nutrition, Physical. Circulation. 2006;113(6):898-918.

18. Lyon M, Bashian C, Sheck C, et al. Outcomes following laparoscopic Roux-en-Y gastric bypass (LRYGB)vary by sex: Analysis of 83,059 women and men with morbid obesity. Am J Surg. 2019;217(6):1019-1024.

19. Weidner G. Why do men get more heart disease than women? An international perspective. J Am Coll Health Assoc. 2000; 48(6):291-294.

20. Roddy E, Choi HK. Epidemiology of gout. Rheum Dis Clin North Am 2014;40(2):155-175.

21. Kautzky-Willer A, Harreiter J, Pacini G. Sex and gender differences in risk, pathophysiology and complications of type 2 diabetes mellitus. Endocr Rev. 2016;37(1): 278-316.

22. Malik VS, Pan A, Willett WC, et al. Sugar-sweetened beverages and weight gain in children and adults: A systematic review and meta-analysis. Am J Clin Nutr. 2013;98(4):1084-1102.

23. Juraschek SP, Miller ER, Gelber AC. Body mass index, obesity, and prevalent gout in the United States in 1988-1994 and 2007-2010. Arthritis Care Res. 2013;65(1):127-132.

24. Sun H, Tang H, Jiang S, et al. Gender and metabolic differences of gallstone diseases. World J Gastroenterol. 2009;15(15):1886-1991.

25. Stinton LM, Shaffer EA. Epidemiology of gallbladder disease: Cholelithiasis and cancer. Gut Liver. 2012;6(2):172-187

26. Lovejoy JC. The influence of sex hormones on obesity across the female life span. J Women's Heal. 1998;7(10):1247-1256.

27. Asanuma K, Iijima K, Shimosegawa T. Gender difference in gastroesophageal reflux diseases. World J Gastroenterol. 2016;22(5):1800 1810

28. Mahli A, Hellerbrand C. Alcohol and Obesity: A Dangerous Association for Fatty Liver Disease. Dig Dis. 2016;34(Suppl 1):32-39.

29. Rosenberger PH, Henderson KE, Grilo CM. Psychiatric disorder comorbidity and association with eating disorders in bariatric surgery patients: A cross-sectional study using structured interview-based diagnosis. J Clin Psychiatry. 2006;67(7):1080-1085.

30. Shimizu I, Kamochi M, Yoshikawa H, et al. Gender Difference in Alcoholic Liver Disease. In: Trends in Alcoholic Liver Disease Research. Clinical and Scientific Aspects. 2012.

31. Diehl AM. Obesity and alcoholic liver disease. 2004

32. Xu J, Lai KKY, Verlinsky A, et al. Synergistic steatohepatitis by moderate obesity and alcohol in mice despite increased adiponectin and p-AMPK. $J$ Hepatol. 2011;55(3):673-682. 